\title{
Malignant Transformation in Glioma Steered by an Angiogenic Switch: Defining a Role for Bone Marrow-Derived Cells
}

\author{
Raymond $\mathrm{Xu}^{1}$, David Pisapia ${ }^{2}$, Jeffrey P. Greenfield ${ }^{3}$ \\ 1. Neurological Surgery, Weill Cornell Medical College 2. Pathology, Weill Cornell Medical College 3. \\ Neurological Surgery, New York-Presbyterian/Weill Cornell Medical College
}

$\square$ Corresponding author: Raymond Xu, xuraymond92@gmail.com

Disclosures can be found in Additional Information at the end of the article

\section{Abstract}

Low-grade gliomas, such as pilocytic astrocytoma and subependymoma, are often characterized as benign tumors due to their relative circumscription radiologically and typically nonaggressive biologic behavior. In contrast, low-grades that are by their nature diffusely infiltrative, such as diffuse astrocytomas and oligodendrogliomas, have the potential to transform into malignant high-grade counterparts and, given sufficient time, invariably do so. These high-grade gliomas carry very poor prognoses and are largely incurable, warranting a closer look at what causes this adverse transition. A key characteristic that distinguishes lowand high-grade gliomas is neovascularization: it is absent in low-grade gliomas, but prolific in high-grade gliomas, providing the tumor with ample blood supply for exponential growth. It has been well described in the literature that bone marrow-derived cells (BMDCs) may contribute to the angiogenic switch that is responsible for malignant transformation of lowgrade gliomas. In this review, we will summarize the current literature on BMDCs and their known contribution to angiogenesis-associated tumor growth in gliomas.

Received 12/08/2015 Review began 12/17/2015 Review ended 12/29/2015 Published 01/27/2016

C) Copyright 2016

Xu et al. This is an open access article distributed under the terms of the Creative Commons Attribution License CC-BY 3.0., which permits unrestricted use, distribution, and reproduction in any medium, provided the original author and source are credited.
Categories: Neurosurgery, Oncology

Keywords: glioma, low-grade glioma, gbm, recurrent glioblastoma, high-grade glioma, malignant transformation, angiogenesis, neovascularization, angiogenic switch, bone marrow-derived cells

\section{Introduction And Background}

\section{Current standard of treatment for low-grade gliomas}

Gliomas exist as a spectrum of low- and high-grade states and are classified as Grade I, II, III, or IV, according to the 2007 World Health Organization (WHO). Low-grade infiltrating gliomas are currently assigned a WHO grade of II and are characterized by relatively low cellularity, mild to moderate nuclear pleomorphism, a low proliferative index, and the absence of microvascular proliferation that often correlates with non-enhancing radiological imaging. The median survival projection and 10-year survival rate for low-grade diffuse fibrillary astrocytomas are 4.7 years and $17 \%$, respectively, while oligodendrogliomas confer a slightly better prognosis with 7.1 years and $33 \%$ survival [1]. The management of low-grade gliomas has been a challenging topic in clinical neuro-oncology and is contingent on numerous factors, including clinical presentation, imaging characteristics, clinician opinion and experience, and patient goals [2]. Although gross total resection is the ideal treatment for low-grade tumors amenable to surgery, this option is not always attainable and is based upon the intracranial location of the mass and possible morbidity associated with a resection. Gross total resection demonstrates the greatest five-year overall survival rate at $63 \%$ compared to a $27 \%$ rate with subtotal resection 
[3]. Adjuvant therapies, such as radiation therapy and chemotherapy, are approached with similar prudence, given the toxicity of such treatments. While they may remain indolent for years, Grade II gliomas possess the potential to progress to their Grade III and IV malignant high-grade counterparts, which are aggressive and invasive. The most ominous of these tumors, glioblastoma (GBM), carries a dismal prognosis of an 18-month median survival even with the most aggressive treatments due to inevitable recurrence. These high-grade astrocytic tumors are highly pleomorphic, present a high degree of proliferation, and are characterized by contrast enhancement on MRI. They are also characterized by necrosis, microvascular proliferation, and hemorrhage, and it is postulated that neovascularization in high-grade gliomas may provide the tumor with the blood supply necessary for further growth and proliferation. It has been hypothesized that low-grade tumors encounter a phenomenon known as the angiogenic switch that permits rapid progression and transformation toward malignant high-grade glioma [4].

\section{Review}

\section{Low-grade to high-grade transition characterized by neovascularization}

The link between tumor growth and invasiveness, and angiogenesis has been well-documented [5]. It is logical that the ability of solid tumors to recruit neovasculature would be a ratelimiting step in tumor progression because, as with all cell types, growth depends upon nutrients provided by circulating blood. The spectrum of low- to high-grade gliomas may be a perfect proof of this concept, as neovascularization is absent in clinically indolent low-grade tumors, but represents a prominent feature of malignant high-grade tumors, including highgrade astrocytoma, oligodendrogliomas, and ependymomas. It has been theorized that the relative lack of vascularization in low-grade tumors confines it to a linear growth model [6], whereas the hypervascularization present in GBM allows for exponential growth and subsequent rapid clinical decline [7]. While neovascularization is a normal process that occurs by way of ischemic stimuli, tumors can hijack the machinery to promote growth and invasiveness, activating the angiogenic switch [8]. In this mechanism, signals, such as vascular endothelial growth factor (VEGF), platelet-derived growth factor (PDGF), and hypoxiainducible factor 1-alpha (HIF-1 $\alpha$ ), are released from glioma stem cells in low oxygen states [9], causing proteases to detach pericytes from existing vessels and forming a weak extracellular matrix around the vessel wall that promotes endothelial cell remodeling [10]. This ultimately promotes not only the branching of existing vessels (angiogenesis) but also the formation of de novo vessels (vasculogenesis). There is evidence that high-grade tumors have transcriptional alterations that correlate with neovascularization, and the upregulation of these genes may also play a role in activating the angiogenic switch. Godard, et al. observed upregulation in several angiogenesis-associated genes, such as VEGF, fibroblast growth factor (FGF), and epidermal growth factor (EGF), in human GBM samples as compared to low-grade astrocytoma [11]. It has also been demonstrated that these factors mobilize a set of BMDCs to the site of neovascularization to further augment the angiogenic effect [12]. Given the limited success of current anti-angiogenic therapies in high-grade gliomas [13], BMDCs offer an additional target to prevent neovascularization and subsequently prevent the malignant transformation of lowgrade gliomas. In order to elucidate potential areas of study, we must first characterize what we already know about BMDCs.

\section{Implication of BMDC contribution in tumors}

BMDCs are a heterogeneous population of cells comprising endothelial (EPCs) and hematopoietic precursor cells (HPCs), mesenchymal stem cells (MSCs), myeloid-derived suppressor cells (MDSCs), Tie-2 expressing monocytes (TEMs), and tumor-associated macrophages (TAMs). While the exact role of each subset has not been precisely defined in the 
context of neovascularization and tumor invasion, it has been shown that BMDCs do in fact help create a niche capable of sustaining tumor growth by breaking down normal structures to promote neovascularization and tumor proliferation. This has been supported by a welldescribed contribution of BMDCs in metastatic disease [14] where bone marrow precursors have been found mobilized and directed to metastatic sites prior to tumor formation, perhaps acting as a primer for tumor invasion. In the brain, mobilization of bone marrow precursors to the tumor has also been demonstrated to promote vasculogenesis in gliomas through a hypoxia-induced mechanism [15]. Again, we revisit the idea that neovascularization is triggered by hypoxic stimuli, occurring most relevantly in the hypoxic tumor environment. Du, et al. showed that release of VEGF and HIF-1 $\alpha$ by tumor cells, in combination with stromal cellderived factor 1 (SDF-1) and C-X-C chemokine receptor type 4 (CXCR-4) receptor activation, spurred mobilization of BMDCs to the invasive tumor front. Specifically, the recruitment of EPCs and myeloid cells to the tumor site induced a pro-angiogenic state [16]. This led to speculation that radiation may play a role in tumor recurrence by way of hypoxia-induced vasculogenesis [17]. Given the significance of neovascularization in malignant transformation, and the reported contribution of BMDCs in angiogenesis, these findings offer further support for the theory that BMDCs play a role in the angiogenic switch thought to cause malignant transformation (Figure 1).

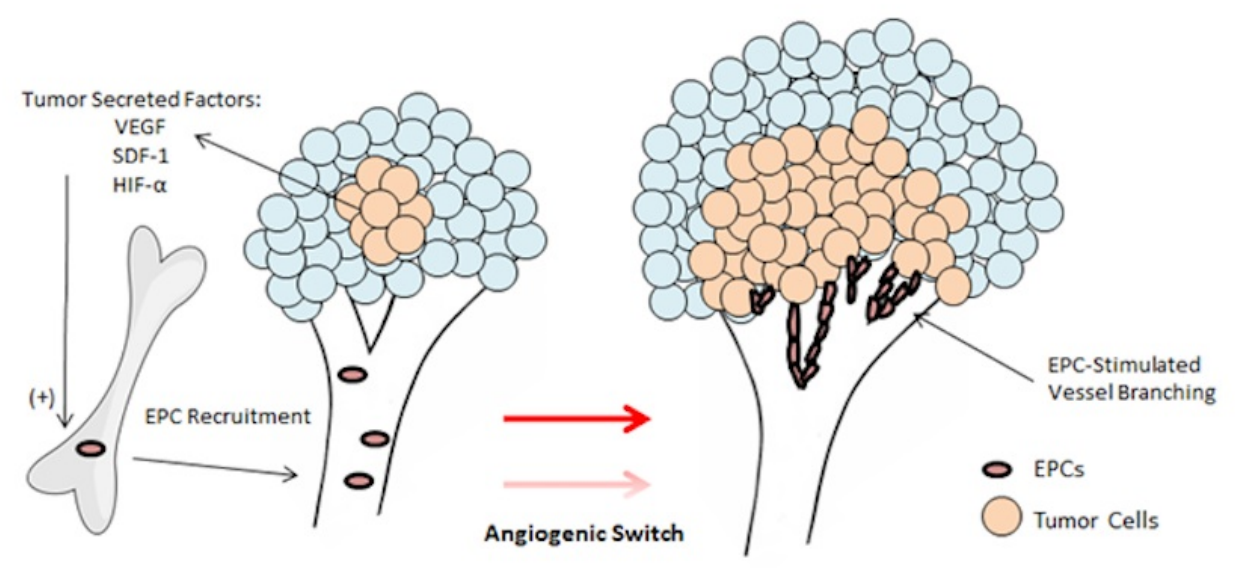

FIGURE 1: Tumor-derived factors recruit endothelial progenitor cells to tumor site, activating the angiogenic switch necessary for malignant transformation

Adapted from Rafii \& Lyden [4].

\section{Clinical implications of BMDC involvement in transformation}

Molecular profiling of various BMDC subsets has helped to identify and characterize the populations of cells involved in clinical incidences of malignant progression. Quantifying these populations holds substantial therapeutic implications. EPCs, which have been phenotypically described as $\mathrm{CD} 133^{+}$and VEGFR $2^{+}$cells, have been found in the peripheral blood of malignant astrocytoma patients [18]. Interestingly, the presence of this population of precursor cells was significantly increased in brain tumor patients compared to normal controls, and also correlated with tumor grade and predicted survival [19-20]. This finding was congruous with previous animal studies using a PDGF-driven murine model of GBM that closely models a lowgrade to high-grade transition. In those studies, specific populations of BMDCs were found in 
the peripheral blood of mice with high-grade tumors but not in mice bearing low-grade tumors [20]. Clinically, a CD $11 b^{+}$MDSC population has demonstrated the capability to distinguish between low and high-grade tumors (unpublished data). Immunofluorescent staining indicated both highly prevalent CD11 $\mathrm{b}^{+}$cells in human GBM and aberrant (CD144 ${ }^{+}$-labeled) vessel formation that was significantly reduced in comparable human Grade II astrocytomas (unpublished data). As previously stated, myeloid cells have been known to contribute to a proangiogenic environment, however, the $\mathrm{CD} 11 \mathrm{~b}^{+}$subset also has been widely associated with inflammation and anti-tumor immunosuppression mechanisms involved with malignant gliomas, notably GBM [21], implicating it as a potentially significant factor in malignant progression. Further characterizing BMDC subsets in low-grade glioma patients may provide the necessary biomarkers to measure the potential for tumor angiogenicity and malignancy, ultimately increasing our ability to predict the likelihood of malignant transformation.

\section{Conclusions}

It is apparent that the angiogenic switch plays a crucial role in the malignant transition of lower grade diffusely infiltrating gliomas to their high-grade counterparts, in part by providing the circulating nutrients required for exponential growth. Anti-angiogenic therapies more directly targeting vascular elements themselves have shown limited clinical efficacy; thus, our attention has shifted to a population of bone marrow-derived precursor cells that have been demonstrated to participate in neovascularization, tumor growth, and tumor invasiveness. While BMDCs are likely not the only contributing element to malignant transformation, targeting their recruitment to tumor sites has demonstrated efficacy in reducing tumor growth in animal models of other tumor types [22]. Furthermore, BMDCs are both practical and feasible as a therapeutic target and prognostic marker. Monitoring BMDC-related biomarkers in the peripheral blood for evidence of transformation may represent an improved prognostic tool over current methods, such as serial imaging, and may ultimately be less costly and easier to perform on a routine basis. Also, given that they are present in the peripheral blood, BMDCs would be particularly accessible as a potential therapeutic target, unprotected by the bloodbrain barrier. Ultimately, improving our understanding of BMDCs in the context of neovascularization may have significant implications on improving clinical care of low-grade diffusely infiltrating gliomas and may provide the key target, that when leveraged properly, could prevent or delay the inevitable transformation to the high-grade, and ultimately fatal, phase of the disease.

\section{Additional Information Disclosures}

Conflicts of interest: In compliance with the ICMJE uniform disclosure form, all authors declare the following: Payment/services info: All authors have declared that no financial support was received from any organization for the submitted work. Financial relationships: All authors have declared that they have no financial relationships at present or within the previous three years with any organizations that might have an interest in the submitted work. Other relationships: All authors have declared that there are no other relationships or activities that could appear to have influenced the submitted work.

\section{References}

1. Cahill DP, Sloan AE, Nahed BV, Aldape KD, Louis DN, Ryken TC, Kalkanis SN, Olson JJ: The role of neuropathology in the management of patients with diffuse low grade glioma: A systematic review and evidence-based clinical practice guideline. J Neurooncol. 2015, 125:531-49. 10.1007/s11060-015-1909-8

2. Whittle IR: The dilemma of low grade glioma. J Neurol Neurosurg Psychiatry. 2004, 75: ii31ii36. 10.1136/jnnp.2004.040501 
3. Prabhu VC, Khaldi A, Barton KP, Melian E, Schneck MJ, Primeau MJ, Lee JM: Management of diffuse low-grade cerebral gliomas. Neurol Clin. 2010, 28:1037-59. 10.1016/j.ncl.2010.03.022

4. Rafii S, Lyden D: Cancer. A few to flip the angiogenic switch . Science. 2008, 319:163-64. 10.1126/science. 1153615

5. Folkman J: Role of angiogenesis in tumor growth and metastasis . Semin Oncol. 2002, 29:1518. 10.1053/sonc.2002.37263

6. Mandonnet E, Delattre JY, Tanguy ML, Swanson KR, Carpentier AF, Duffau H, Cornu P, Van Effenterre R, Alvord EC Jr, Capelle L: Continuous growth of mean tumor diameter in a subset of grade II gliomas. Ann Neurol. 2003, 53:524-28. 10.1002/ana.10528

7. Rees J, Watt H, Jäger HR, Benton C, Tozer D, Tofts P, Waldman A: Volumes and growth rates of untreated adult low-grade gliomas indicate risk of early malignant transformation. Eur J Radiol. 2009, 72:54-64. 10.1016/j.ejrad.2008.06.013

8. Bergers G, Benjamin LE: Tumorigenesis and the angiogenic switch. Nat Rev Cancer. 2003, 3:401-10. 10.1038/nrc1093

9. Bulnes S, Bengoetxea H, Ortuzar N, Argandoña EG, Garcia-Blanco A, Rico-Barrio I, Lafuente JV: Angiogenic signalling pathways altered in gliomas: selection mechanisms for more aggressive neoplastic subpopulations with invasive phenotype. J Signal Transduct. 2012, 2012:597915. 10.1155/2012/597915

10. Bergers G, Song S: The role of pericytes in blood-vessel formation and maintenance . Neuro Oncol. 2005, 7:452-64. 10.1215/s1152851705000232

11. Godard S, Getz G, Delorenzi M, Farmer P, Kobayashi H, Desbaillets I, Nozaki M, Diserens AC, Hamou MF, Dietrich PY, Regli L, Janzer RC, Bucher P, Stupp R, de Tribolet N, Domany E, Hegi ME: Classification of human astrocytic gliomas on the basis of gene expression: a correlated group of genes with angiogenic activity emerges as a strong predictor of subtypes. Cancer Res. 2003, 63:6613-25.

12. Lyden D, Hattori K, Dias S, Costa C, Blaikie P, Butros L, Chadburn A, Heissig B, Marks W, Witte L, Wu Y, Hicklin D, Zhu Z, Hackett NR, Crystal RG, Moore MA, Hajjar KA, Manova K, Benezra R, Rafii S: Impaired recruitment of bone-marrow-derived endothelial and hematopoietic precursor cells blocks tumor angiogenesis and growth. Nat Med. 2001, 7:1194201. 10.1038/nm1101-1194

13. Vasudev NS, Reynolds AR: Anti-angiogenic therapy for cancer: current progress, unresolved questions and future directions. Angiogenesis. 2014, 17:471-94. 10.1007/s10456-014-9420-y

14. Wels J, Kaplan RN, Rafii S, Lyden D: Migratory neighbors and distant invaders: tumorassociated niche cells. Genes Dev. 2008, 22:559-74. 10.1101/gad.1636908

15. Du R, Lu KV, Petritsch C, Liu P, Ganss R, Passegué E, Song H, Vandenberg S, Johnson RS, Werb Z, Bergers G: HIF1alpha induces the recruitment of bone marrow-derived vascular modulatory cells to regulate tumor angiogenesis and invasion. Cancer Cell. 2008, 13:206-20. 10.1016/j.ccr.2008.01.034

16. Urbich C, Dimmeler S: Endothelial progenitor cells: characterization and role in vascular biology. Circ Res. 2004, 95:343-53. 10.1161/01.res.0000137877.89448.78

17. Kioi M, Vogel H, Schultz G, Hoffman RM, Harsh GR, Brown JM: Inhibition of vasculogenesis, but not angiogenesis, prevents the recurrence of glioblastoma after irradiation in mice. J Clin Invest. 2010, 120:694-705. 10.1172/JCI40283

18. Chantrain CF, Feron O, Marbaix E, DeClerck YA: Bone marrow microenvironment and tumor progression. Cancer Microenviron. 2008, 1:23-35. 10.1007/s12307-008-0010-7

19. Rafat N, Beck GCh, Schulte J, Tuettenberg J, Vajkoczy P: Circulating endothelial progenitor cells in malignant gliomas. J Neurosurg. 2010, 112:43-49. 10.3171/2009.5.JNS081074

20. Greenfield JP, Jin DK, Young LM, Christos PJ, Abrey L, Rafii S, Gutin PH: Surrogate markers predict angiogenic potential and survival in patients with glioblastoma multiforme. Neurosurgery. 2009, 64:819-26. 10.1227/01.NEU.0000343742.06625.DB

21. Chae M, Peterson TE, Balgeman A, Chen S, Zhang L, Renner DN, Johnson AJ, Parney IF: Increasing glioma-associated monocytes leads to increased intratumoral and systemic myeloid-derived suppressor cells in a murine model. Neuro Oncol. 2015, 17:978-91. 10.1093/neuonc/nou343

22. Chen Y, Gou X, Kong DK, Wang X, Wang J, Chen Z, Huang C, Zhou J: EMMPRIN regulates tumor growth and metastasis by recruiting bone marrow-derived cells through paracrine signaling of SDF-1 and VEGF. Oncotarget. 2015, 6:32575-85. 10.18632/oncotarget.5331 